\title{
Analytic Solution of the Pion-Laser Model
}

\author{
T. Csörgö ${ }^{1,2}$ and J. Zimányi ${ }^{1}$ \\ ${ }^{1}$ Department of Physics, Columbia University, $538 \mathrm{~W}$ 120-th Street, New York, NY 10027 \\ ${ }^{2}$ MTA KFKI RMKI, H-1525 Budapest 114. POB. 49, Hungary
}

(September 23, 2018)

\begin{abstract}
Brooding over bosons, wave packets and Bose - Einstein correlations, we find that a generalization of the pion-laser model for the case of overlapping wave-packets is analytically solvable with complete $n$-particle symmetrization. Multi-boson correlations generate momentum-dependent radius and intercept parameters even for static sources. Explicit multiplicity dependence of exclusive correlations and spectra is found. The HBT radii are reduced for low values and enlargened for high values of the mean momentum in the rare gas limiting case.
\end{abstract}

25.75.gz,25.75.-q,03.65.-w,05.30.Jp

Introduction. The study of the statistical properties of quantum systems has a long history with important recent developments. In high energy physics, quantum statistical correlations are studied in order to infer the space-time dimensions of the elementary particle reactions. In high energy heavy ion collisions hundreds of bosons are created in the present CERN SPS reactions when $P b+P b$ reactions are measured at $160 \mathrm{AGeV}$ laboratory bombarding energy. At the RHIC accelerator, to be completed by 1999, thousands of pions could be produced in a unit rapidity interval [1.21. If the number of pions in a unit value of phase-space is large enough these bosons may condense into the same quantum state and a pion laser could be created [3]. Similarly to this process, when a large number of bosonic atoms are collected in a magnetic trap and cooled down to increase their density in phase-space, the bosonic nature of the atoms reveals itself in the formation of a Bose-Einstein condensate [4], a macroscopic quantum state. Such a condensation mechanism may provide the key to the formation of atomic lasers in condensed matter physics and to the formation of pion lasers in high energy particle and heavy ion physics, reviewed recently in refs 河 7 .

The density matrix of a generic quantum mechanical system is

$$
\hat{\rho}=\sum_{n=0}^{\infty} p_{n} \hat{\rho}_{n}
$$

Here the index $n$ characterizes sub-systems with particle number fixed to $n$, the multiplicity distribution is prescribed by the set of $\left\{p_{n}\right\}_{n=0}^{\infty}$, normalized as $\sum_{n=0}^{\infty} p_{n}=1$. The density matrixes are normalized as $\operatorname{Tr} \hat{\rho}=1$ and $\operatorname{Tr} \hat{\rho}_{n}=1$, where

$$
\hat{\rho}_{n}=\int d \alpha_{1} \ldots d \alpha_{n} \rho_{n}\left(\alpha_{1}, \ldots, \alpha_{n}\right)\left|\alpha_{1}, \ldots, \alpha_{n}\right\rangle\left\langle\alpha_{1}, \ldots, \alpha_{n}\right|
$$

and the states $\left|\alpha_{1}, \ldots, \alpha_{n}\right\rangle$ denote properly normalized $n$-particle wave-packet boson states.

A wave packet creation operator is

$$
\alpha_{i}^{\dagger}=\int \frac{d^{3} \mathbf{p}}{\left(\pi \sigma^{2}\right)^{\frac{3}{4}}} \mathrm{e}^{-\frac{\left(\mathbf{p}-\pi_{i}\right)^{2}}{2 \sigma_{i}^{2}}-i \xi_{i}\left(\mathbf{p}-\pi_{i}\right)+i \omega(\mathbf{p})\left(t-t_{i}\right)} \hat{a}^{\dagger}(\mathbf{p}),
$$

where $\alpha_{i}=\left(\xi_{i}, \pi_{i}, \sigma_{i}, t_{i}\right)$ refers to the parameters of the wave packet $i$ : the center in space, in momentum space, the width in momentum space and the production time, respectively. For simplicity we assume that all the wave packets are emitted at the same instant and with the same width, $\alpha_{i}=\left(\pi_{i}, \xi_{i}, \sigma, t_{0}\right)$.

The $n$ boson states, normalized to unity, are given as

$$
\left|\alpha_{1}, \ldots, \alpha_{n}\right\rangle=\left(\sum_{\sigma^{(n)}} \prod_{i=1}^{n}\left\langle\alpha_{i} \mid \alpha_{\sigma_{i}}\right\rangle\right)^{-\frac{1}{2}} \alpha_{n}^{\dagger} \ldots \alpha_{1}^{\dagger}|0\rangle .
$$


Here $\sigma^{(n)}$ denotes the set of all the permutations of the indexes $\{1,2, \ldots, n\}$ and the subscript sized $\sigma_{i}$ denotes the index that replaces the index ${ }_{i}$ in a given permutation from $\sigma^{(n)}$.

Solution for a New Type of Density Matrix There is one special density matrix, for which one can overcome the difficulty, related to the non-vanishing overlap of many hundreds of wave-packets, even in an explicit analytical manner. Namely, if one assumes, that we have a system, in which the emission probability of a boson is increased if there is an other emission in the vicinity:

$$
\rho_{n}\left(\alpha_{1}, \ldots, \alpha_{n}\right)=\frac{1}{\mathcal{N}(n)} \prod_{i=1}^{n} \rho_{1}\left(\alpha_{i}\right)\left(\sum_{\sigma^{(n)}} \prod_{k=1}^{n}\left\langle\alpha_{k} \mid \alpha_{\sigma_{k}}\right\rangle\right)
$$

The coefficient of proportionality, $\mathcal{N}(n)$, can be determined from the normalization condition. The density matrix of eq. (5) describes a quantum-mechanical wave-packet system with induced emission, and the amount of the induced emission is controlled by the overlap of the $n$ wave-packets [8], yielding a weight in the range of [1, $n !$ ]. Although it is very difficult numerically to operate with such a wildly fluctuating weight, we were able to reduce the problem [8] to an already discovered "ring" - algebra of permanents for plane-wave outgoing states [3].

For the sake of simplicity we assume a non-relativistic, non-expanding static source at rest in the frame where the calculations are performed.

$$
\begin{aligned}
& \rho_{1}(\alpha)=\rho_{x}(\xi) \rho_{p}(\pi) \delta\left(t-t_{0}\right), \\
& \rho_{x}(\xi)=\frac{1}{\left(2 \pi R^{2}\right)^{\frac{3}{2}}} \exp \left(-\xi^{2} /\left(2 R^{2}\right)\right), \\
& \rho_{p}(\pi)=\frac{1}{(2 \pi m T)^{\frac{3}{2}}} \exp \left(-\pi^{2} /(2 m T)\right),
\end{aligned}
$$

and a Poisson multiplicity distribution $p_{n}^{(0)}$ for the case when the Bose-Einstein effects are negligible:

$$
p_{n}^{(0)}=\frac{n_{0}^{n}}{n !} \exp \left(-n_{0}\right)
$$

This corresponds to the very rare gas limit, and completes the specification of the model. The plane-wave model, to which the multi-particle wave-packet model was reduced in ref. [8], can be further simplified [8] to a set of recurrence relations with the help of the so-called "ring-algebra" discovered first by S. Pratt in ref. [3]. The probability of finding events with multiplicity $n$, as well as the single-particle and the two-particle momentum distribution in such events is given as

$$
\begin{aligned}
p_{n} & =\omega_{n}\left(\sum_{k=0}^{\infty} \omega_{k}\right)^{-1}, \\
N_{1}^{(n)}\left(\mathbf{k}_{1}\right) & =\sum_{i=1}^{n} \frac{\omega_{n-i}}{\omega_{n}} G_{i}(1,1), \\
N_{2}^{(n)}\left(\mathbf{k}_{1}, \mathbf{k}_{2}\right) & =\sum_{l=2}^{n} \sum_{m=1}^{l-1} \frac{\omega_{n-l}}{\omega_{n}}\left[G_{m}(1,1) G_{l-m}(2,2)+\right. \\
& \left.+G_{m}(1,2) G_{l-m}(2,1)\right],
\end{aligned}
$$

where $\omega_{n}=p_{n} / p_{0}$ and

$$
G_{n}(i, j)=n_{0}^{n} h_{n} \exp \left(-a_{n}\left(\mathbf{k}_{i}^{2}+\mathbf{k}_{j}^{2}\right)+g_{n} \mathbf{k}_{i} \mathbf{k}_{j}\right) .
$$

Averaging over the multiplicity distribution $p_{n}$ yields the inclusive spectra as 


$$
\begin{aligned}
G(1,2) & =\sum_{n=1}^{\infty} G_{n}(1,2), \\
N_{1}\left(\mathbf{k}_{1}\right) & =\sum_{n=1}^{\infty} p_{n} N_{1}^{(n)}\left(\mathbf{k}_{1}\right)=G(1,1), \\
N_{2}\left(\mathbf{k}_{1}, \mathbf{k}_{2}\right) & =G(1,1) G(2,2)+G(1,2) G(2,1) .
\end{aligned}
$$

An auxiliary quantity is introduced as

$$
C_{n}=\frac{1}{n} \int d^{3} \mathbf{k}_{1} G_{n}(1,1)=h_{n} \frac{n_{0}^{n}}{n}\left(\frac{\pi}{2 a_{n}-g_{n}}\right)^{\frac{3}{2}} .
$$

With the help of the notation

$$
\sigma_{T}^{2}=\sigma^{2}+2 m T, \quad R_{e}^{2}=R^{2}+\frac{m T}{\sigma^{2} \sigma_{T}^{2}},
$$

the recurrence relations that correspond to the solution of the ring-algebra [9,3] are obtained [8] for the case of the multi-particle wave-packet model. These correspond to the pion laser model of S. Pratt when a replacement $R \rightarrow R_{e}$ and $T \rightarrow T_{e}=\sigma_{T}^{2} /(2 m)$ is performed.

Let us introduce the following auxiliary quantities:

$$
\gamma_{ \pm}=\frac{1}{2}(1+x \pm \sqrt{1+2 x}) \quad x=R_{e}^{2} \sigma_{T}^{2}
$$

The general analytical solution of the model is given through the generating function of the multiplicity distribution $p_{n}$

$$
G(z)=\sum_{n=0}^{\infty} p_{n} z^{n}=\exp \left(\sum_{n=1}^{\infty} C_{n}\left(z^{n}-1\right)\right)
$$

where $C_{n}$ is

$$
C_{n}=\frac{n_{0}^{n}}{n}\left[\gamma_{+}^{\frac{n}{2}}-\gamma_{-}^{\frac{n}{2}}\right]^{-3}
$$

together with the general analytic solution for the functions $G_{n}(1,2)$ :

$$
\begin{aligned}
G_{n}(1,2) & =j_{n} \mathrm{e}^{-\frac{b_{n}}{2}\left[\left(\gamma_{+}^{\frac{n}{2}} \mathbf{k}_{1}-\gamma_{-}^{\frac{n}{2}} \mathbf{k}_{2}\right)^{2}+\left(\gamma_{+}^{\frac{n}{2}} \mathbf{k}_{2}-\gamma_{-}^{\frac{n}{2}} \mathbf{k}_{1}\right)^{2}\right],} \\
j_{n} & =n_{0}^{n}\left[\frac{b_{n}}{\pi}\right]^{\frac{3}{2}} \quad b_{n}=\frac{1}{\sigma_{T}^{2}} \frac{\gamma_{+}-\gamma_{-}}{\gamma_{+}^{n}-\gamma_{-}^{n}}
\end{aligned}
$$

The detailed proof that the analytic solution to the multi-particle wave-packet model is indeed given by the above equations is described in ref. [8].

The representation of eq. (18) indicates that the quantities $C_{n}$-s are the so called combinants [11 13] of the probability distribution of $p_{n}$ and in our case their explicit form is known for any set of model parameters, as given by eqs. 19, 17, 16). The resulting multiplicity generating function does not correspond to discrete probability generating functions in ref [14], we have found a new type of probability generating functions.

One can prove [8], that the mean multiplicity $\langle n\rangle=\sum_{n=1}^{\infty} n p_{n}=\sum_{i=1}^{\infty} i C_{i}$. The large $n$ behavior of $n C_{n}$ depends on the ratio of $n_{0} / \gamma_{+}^{\frac{3}{2}}$, since for large values of $n$, we always have $\left(\gamma_{-} / \gamma_{+}\right)^{\frac{n}{2}}<<1$. The critical value of $n_{0}$ is

$$
n_{c}=\gamma_{+}^{\frac{3}{2}}=\left[\frac{1+x+\sqrt{1+2 x}}{2}\right]^{\frac{3}{2}} .
$$


If $n_{0}<n_{c}$, one finds $\lim _{n \rightarrow \infty} n C_{n}=0$ and $\langle n\rangle<\infty$, if $n_{0}>n_{c}$ one obtains $\lim _{n \rightarrow \infty} n C_{n}=\infty$ and $\langle n\rangle=\infty$, finally, if $n_{0}=n_{c}$ one finds $\lim _{n \rightarrow \infty} n C_{n}=1$ and $\langle n\rangle=\infty$. The divergence of the mean multiplicity $\langle n\rangle$ is related to condensation of the wave-packets to the wave-packet state with $\pi=0$, i.e. to the wave-packet state with zero mean momentum [8], if $n_{0} \geq n_{c}$.

The multiplicity distribution of eq. (18) is studied at greater length in ref. [8].

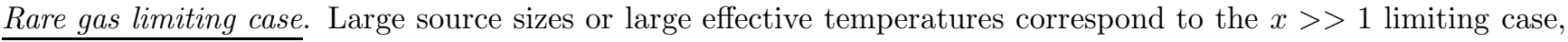
where the general analytical solution of the model, presented above, becomes particularly simple and the exclusive and inclusive spectra and correlation functions can be obtained analytically to leading order in $1 / x<<1$. From eq. (11) one obtains that

$$
\begin{aligned}
G_{n}(1,2) & =j_{n} \exp \left[-\frac{n}{2 \sigma_{T}^{2}}\left(\mathbf{k}_{1}^{2}+\mathbf{k}_{2}^{2}\right)-\frac{R_{e}^{2}}{2 n} \mathbf{\Delta} \mathbf{k}^{2}\right], \\
j_{n} & =\frac{n^{5 / 2} C_{n}}{\left(\pi \sigma_{T}^{2}\right)^{\frac{3}{2}}}, \quad C_{n}=\frac{n_{0}^{n}}{n^{4}}\left(\frac{2}{x}\right)^{\frac{3}{2}(n-1)},
\end{aligned}
$$

where $\Delta \mathbf{k}=\mathbf{k}_{1}-\mathbf{k}_{2}$. We can see from eq. (24), that the higher order corrections will contribute to the observables with reduced effective temperatures and reduced effective radii. Eq. (24) indicates, that the leading order result for the combinants in the $x>>1$ limiting case is $C_{1}$ with the first sub-leading correction given by $C_{2}$. Thus, the probability distribution can be considered in the rare gas limiting case as a Poisson distribution of particle singlets with a sub-leading correction that yields a convolution of Poisson-distributed doublets.

The very rare gas limiting case corresponds to keeping only the leading $n=1$ order terms in the above equations. The multiplicity distribution is a Poisson distribution with $\langle n\rangle=n_{0}$ and no influence from stimulated emission. The momentum distribution is a static Boltzmann distribution, and the exclusive and inclusive momentum distributions coincide [8]. The leading order two-particle Bose-Einstein correlation function is a static Gaussian correlation function with a constant intercept parameter of $\lambda=1$ and with a momentum - independent radius parameter of $R_{*}=R_{e}$ [8].

This wave-packet model exhibits a lasing behavior in the very dense Bose-gas limit, which corresponds to an optically coherent behavior, characterized by a vanishing enhancement of the two-particle intensity correlations at low momentum, $C\left(\mathbf{k}_{1}, \mathbf{k}_{2}\right)=1$, a case which is described in greater details in ref. [8].

The probability generating function yields the following leading order multiplicity distribution:

$$
p_{n}=\frac{n_{0}^{n}}{n !} \exp \left(-n_{0}\right)\left[1+\frac{n(n-1)-n_{0}^{2}}{2(2 x)^{\frac{3}{2}}}\right] .
$$

The mean multiplicity, the factorial cumulant moments of the multiplicity distribution, the inclusive and exclusive momentum distributions were evaluated by keeping only the leading order terms in $1 / x$ in ref. [8]. The two-particle exclusive correlation functions can also be evaluated by applying a Gaussian approximation to the leading order corrections in the $x>>1$ limiting case:

$$
\begin{aligned}
C_{2}^{(n)}\left(\mathbf{k}_{1}, \mathbf{k}_{2}\right) & =\frac{n^{2}}{n(n-1)} \frac{N_{2}^{(n)}\left(\mathbf{k}_{1}, \mathbf{k}_{2}\right)}{N_{1}^{(n)}\left(\mathbf{k}_{1}\right) N_{1}^{(n)}\left(\mathbf{k}_{2}\right)} \\
& =1+\lambda_{\mathbf{K}} \exp \left(-R_{\mathbf{K}, s}^{2} \boldsymbol{\Delta} \mathbf{k}_{s}^{2}-R_{\mathbf{K}, o}^{2} \Delta \mathbf{k}_{o}^{2}\right),
\end{aligned}
$$

where $\mathbf{K}=0.5\left(\mathbf{k}_{1}+\mathbf{k}_{2}\right)$, the side and outwards directions are introduced utilizing the spherical symmetry of the source as $\Delta \mathbf{k}_{s}=\Delta \mathbf{k}-\mathbf{K}(\Delta \mathbf{k} \cdot \mathbf{K}) /(\mathbf{K} \cdot \mathbf{K})$ and $\Delta \mathbf{k}_{o}=\mathbf{K}(\Delta \mathbf{k} \cdot \mathbf{K}) /(\mathbf{K} \cdot \mathbf{K})$, similarly to refs. 15. The mean-momentum dependent intercept and radius parameters are

$$
\lambda_{\mathbf{K}}=1+\frac{2}{(2 x)^{\frac{3}{2}}}\left[1-2^{(5 / 2)} \exp \left(-\frac{\mathbf{K}^{2}}{\sigma_{T}^{2}}\right)\right]
$$




$$
\begin{aligned}
R_{\mathbf{K}, s}^{2}=R_{e}^{2}+ & \frac{1}{(2 x)^{\frac{3}{2}}}\left[R_{e}^{2}-\sqrt{2} \exp \left(-\frac{\mathbf{K}^{2}}{\sigma_{T}^{2}}\right) \times\right. \\
& \left.\times\left((n+2) R_{e}^{2}+\frac{2}{\sigma_{T}^{2}}\right)\right], \\
R_{\mathbf{K}, o}^{2}=R_{\mathbf{K}, s}^{2} & +\frac{n}{x^{\frac{3}{2}}} \frac{\mathbf{K}^{2}}{\sigma_{T}^{4}} \exp \left(-\frac{\mathbf{K}^{2}}{\sigma_{T}^{2}}\right)
\end{aligned}
$$

Thus the symmetrization results in a momentum - dependent intercept parameter $\lambda_{\mathbf{K}}$ that starts from a $\lambda_{\mathbf{K}=0}<1$ value at low momentum and increases with increasing momentum. Already in the first paper about the pion laser model, ref. [3], a reduction of the exact intercept parameter was observed and interpreted as the onset of a coherent behavior in the low momentum modes. First, a partially coherent system is created, characterized by $\lambda_{\mathbf{K}}<1$, and if the density of pions is further increased, one finds a fully developed pion-laser with $\lambda_{\mathbf{K}}=0$, see ref. [8] for analytic considerations.

Observe that the radius parameter at low mean momentum decreases while the radius parameter at high mean momentum increases, as compared to $R_{e}$. The radius parameter of the exclusive correlation function thus becomes momentum-dependent even for static sources! This effect is more pronounced for higher values of the fixed multiplicity $n$, in contrast to the momentum dependence of $\lambda_{\mathbf{K}}$ that is independent of $n$.

Last, but not least, a specific term appears in the two-particle exclusive correlation function, that contributes only to the out direction, which, in case of spherically symmetric sources, may be identified with the direction of the mean momentum 15]. This directional dependence is related only to the direction of the relative momentum as compared to the direction of the mean momentum, and does not violate the assumed spherical symmetry of the boson source. The effect vanishes both at very low or at very high values of the mean momentum $\mathbf{K}$, according to eq. (29). The top, middle and bottom panel of Figure 1 indicates the momentum dependent $\lambda_{\mathbf{K}}$ intercept parameter, the $R_{\mathbf{K}, s}$ and $R_{\mathbf{K}, o}$ radius parameters for a fireball with $R=11 \mathrm{fm}, T=120 \mathrm{MeV}$. The pions are assumed to be described by wave-packets with spatial widths of $\sigma_{x}=2 \mathrm{fm}$, and events with fixed identical pion multiplicity of $n_{\pi}=600$ are selected for the evaluation of the correlation function. For this set of parameters, the enhancement of $\lambda_{\mathbf{K}}, R_{\mathbf{K} \text {,side }}$ and $R_{\mathbf{K} \text {,out }}$ is hardly noticeable at high momentum, but their small decrease at low momentum is clear. One may consider a small cold pionic system with a few large wave-packets only, by inserting $R=4 \mathrm{fm}, T=10 \mathrm{MeV}, n_{\pi}=3$ and $\sigma_{x}=5 \mathrm{fm}$ to eqs. (27-29). This source could correspond to heavy ion collisions in the $30 \mathrm{MeV}$ A energy domain 15] characterized by an effective radius $R_{e}=4.5 \mathrm{fm}$ and effective temperature of $T_{e}=15.6 \mathrm{MeV}$ [15]. In this case, the directional dependence of the radii and the enhancement of the radius parameters at high momentum as compared to $R_{e}$ becomes significant not only analytically but numerically as well.

Highlights : In this Letter a consequent quantum mechanical description of multi-boson systems is presented, using properly normalized projector operators for overlapping multi-particle wave-packet states describing stimulated emission of bosons. Our new analytic result is that multi-boson correlations generate momentum-dependent radius and intercept parameters even for static sources, as well as induce a special directional dependence of the correlation function. The effective radius parameter of the two-particle correlation function is reduced for low values and enlargened for large values of the mean momentum in the rare gas limiting case, as compared to the case when multi-particle symmetrization effects are neglected. For extended, hot and rare gas of a few hundred pions, the reduction of the radius parameters at low momentum is found to be the most apparent effect. The directional dependence of the radius parameters and the enhancement of the radii at high momentum is characteristic for a small, cold pion gas with only a handful of particles in it. These results can be understood qualitatively by an enhancement of the wave-packets in the low momentum modes, due to multi-particle Bose-Einstein symmetrization effects, as the system starts to approach the formation of a laser, characterized by the appearance of partial optical coherence in the low momentum modes. 
Our results explicitly depend on the multiplicity, providing a theoretical basis for event-by-event analysis of heavy ion data.

Acknowledgments : Cs. T. would like to thank M. Gyulassy, S. Hegyi, G. Vahtang and X. N. Wang for stimulating discussions. This work was supported by the NSF - HAS Grant INT 8210278, and by the OTKA Grants No. F4019, W01015107 and T024094, by the USA - HJF grant MAKA 378/93 and by an Advanced Research Award from the Fulbright Foundation.

[1] Proceedings of the Quark Matter'96 conference, (P. Braun-Munzinger, H. J. Specht, R. Stock and H. Stöcker, eds.), Nucl. Phys. A610 (1996), 1c - 565c.

[2] Proceedings of the Strangeness in Hadronic Matter'96 conference (ed. by T. Csörgő, P. Lévai and J. Zimányi) Heavy Ion Physics 4 (1996) 1 - 440.

[3] S. Pratt, Phys. Lett. B 301 (1993) 159.

[4] M. Anderson, J. Enscher, M. Matthews, C. Wieman, E. Cornell, Science, 269 (1995) 198.

[5] B. Lörstad, Int. J. Mod. Phys. A12 (1989) 2861.

[6] W. A. Zajc, in "Particle Production in Highly Excited Matter", NATO ASI series B303 (Plenum Press, 1993, ed. H. Gutbrod and J. Rafelski) 435.

[7] U. Heinz, Nucl. Phys. A610 (1996) 264c.

[8] J. Zimányi and T. Csörgő, CU-TP-818/1997, submitted to Phys. Rev. C.

[9] W. Q. Chao , C. S. Gao, Q.H. Zhang, J. Phys. G. Nucl. Part. Phys. 21 (1995) 847.

[10] T. Csörgö, in preparation

[11] M. Gyulassy and S. K. Kaufmann, Phys. Rev. Lett. 40 (1978) 298; S. K. Kaufmann and M. Gyulassy, J. Phys. A 11 (1978) 1715

[12] S. Hegyi, Phys. Lett. B309 (1993) 443-450

[13] S. Hegyi, Phys. Lett. B318 (1993) 642-647

[14] N. L. Johnson, S. Kotz and A. W. Kemp, Univariate Discrete Distributions, (John Wiley and Sons, second edition, 1992)

[15] T. Csörgő, B. Lörstad and J. Zimányi, Phys. Lett. B338 (1994) 134; J. Helgesson, T. Csörgő, M. Asakawa and B. Lörstad, nucl-th/9506006. 


\section{Multi-Particle Symmetrization Effects}

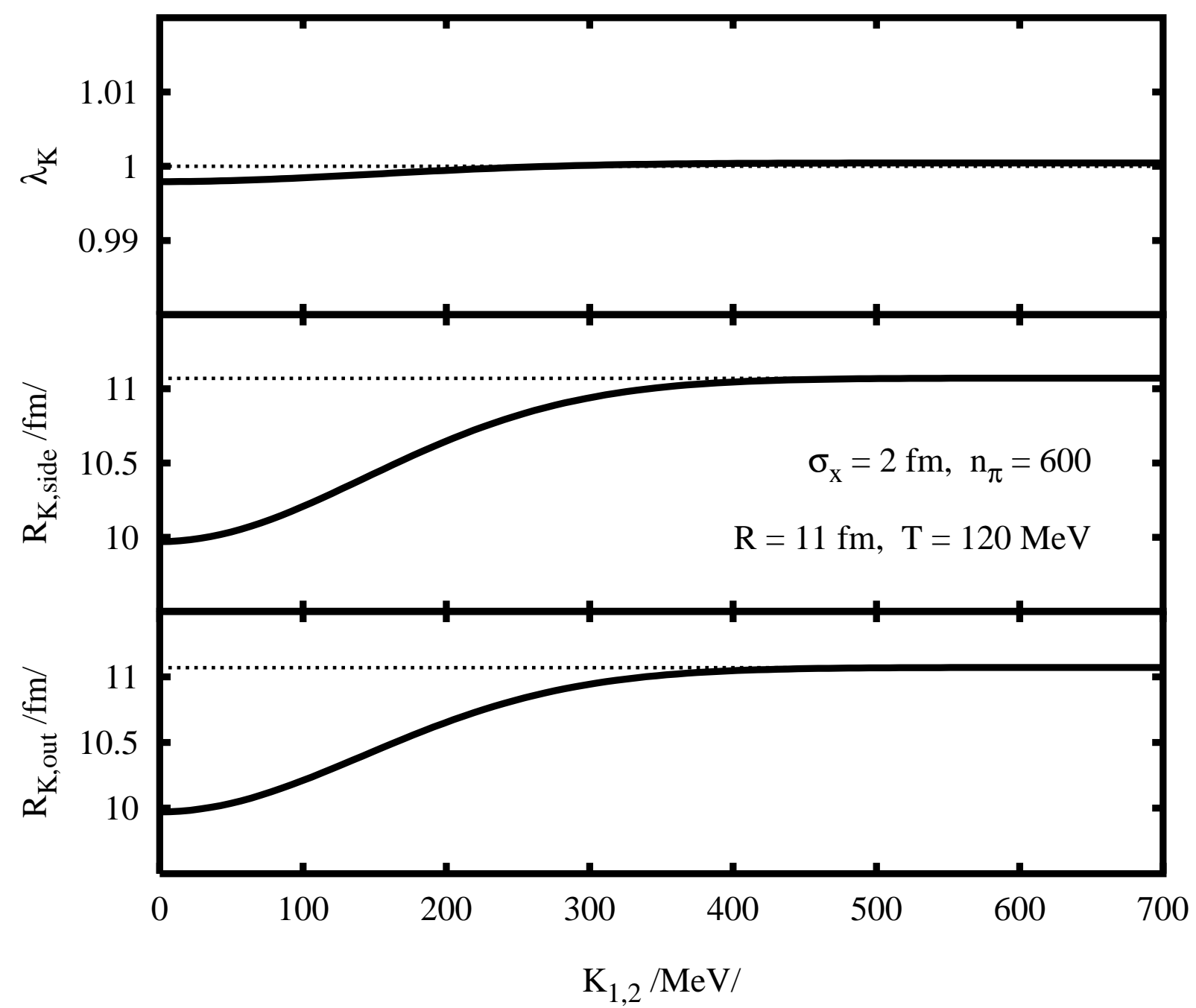

FIG. 1. Multi-particle symmetrization results at low $\mathbf{K}$ in a momentum-dependent reduction of the intercept parameter $\lambda_{\mathbf{K}}$, the side-wards and the outwards radius parameters, $R_{\mathbf{K}, s}$ and $R_{\mathbf{K}, o}$ from their static values of 1 and $R_{e}$, respectively. The enhancement of these parameters at high momentum is hardly noticeable for large and hot systems. 